\title{
Das multiprofessionelle Netzwerk in der Beratung von Geflüchteten
}

\section{Zu den Herausforderungen einer gelingenden Zusammenarbeit und zur Rolle Sozialer Arbeit}

Menschen, die nach Deutschland flüchten, haben gerade in den ersten Jahren einen hohen Beratungsbedarf. Sie benötigen Informationen zu Sprachkursen und Arbeitsmöglichkeiten und suchen nach Antworten auf aufenthaltsrechtliche Fragen. Auch der Familiennachzug ist für viele ein wichtiges Anliegen. Es gibt unterschiedliche Beratungsstellen für Geflüchtete, zudem sind auch Schulen, Behörden, Kanzleien und Ehrenamtliche Teil der Unterstützungsstruktur. Die multiprofessionelle Zusammenarbeit kann dabei einen großen Mehrwert haben, wenn es gelingt, deren Stolpersteine zu umschiffen.

$\mathrm{D}$ ie Beratung von geflüchteten Menschen ist ein seit einigen Jahren stark angewachsenes Handlungsfeld in der Sozialen Arbeit, in dem multiprofessionelle Netzwerkarbeit eine bedeutende Ressource sein kann. Seitdem im Jahr 2005 die Migrationsberatung für Erwachsene (MBE) Teil eines bundesweiten, seitens des Bundesamts für Migration und Flüchtlinge (BAMF) durchgeführten und vom Zuwanderungsgesetz vorgesehenen Integrationsprogramms wurde, zählen auch geflohene Menschen zu ihrer Zielgruppe. Für jugendliche Migrant_innen und Geflüchtete stehen weiterhin die Jugendmigrationsdienste (JMD) ${ }^{1}$ zur Verfügung. Neben den bundesweiten insgesamt

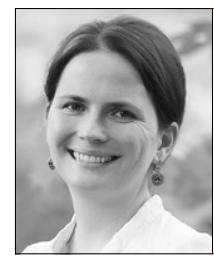

\section{Swantje Penke}

Holzminden, Deutschland

*1983; Dr., seit 2019 Verwalterin der Professur Soziale Arbeit und Diversität an der HAWK in Holzminden, Themenschwerpunkte: Flucht und Migration, Kritische Soziale Arbeit.

swantje.penke@hawk.de

Zusammenfassung In der Migrationsberatung für Geflüchtete stellt ein gut funktionierendes Netzwerk ein bedeutsames Potential in der Unterstützung der Ratsuchenden dar. Neben gegenseitiger Wertschätzung ist es wichtig, die unterschiedlichen institutionellen Rahmungen und Logiken im Netzwerk zu beachten und auf mehreren Ebenen Ressourcen für eine gelingende Netzwerkarbeit zu schaffen. Darin liegt, auch angesichts der prekären Situation der Migrationsberatung, eine besondere Herausforderung für die Soziale Arbeit.

Schlüsselwörter Multiprofessionalität, Netzwerk, Geflüchtete, Asyl, Flucht, Migrationsberatung ca. 1250 MBE-Beratungsstellen (BAGFW 2018) existieren unterschiedliche Landesprogramme, die die Einrichtung von Beratungsstellen für Geflüchtete ermöglichten. In Niedersachsen z. B. ist dies das Programm „Migrationsberatung “ (Ministerium für Soziales, Gesundheit und Gleichstellung 2017), für das jährlich Mittel zur Verfügung gestellt werden, die subsidiär zu den Bundesmitteln MBE/JMD in Anspruch genommen werden sollen. Dabei ist zu beachten, dass eine deutliche Zielgruppendifferenzierung zwischen diesen Programmen existiert: während in der MBE Menschen beraten werden sollen, „, bei denen ein rechtmäßiger und dauerhafter Aufenthalt zu erwarten ist" (BMI 2010, S. 260), ist in der landesfinanzierten Migrationsberatung eine Beratung geflohener Menschen unabhängig von ihrem Aufenthaltsstatus und ihrer aufenthaltsrechtlichen Perspektive möglich. Die MBE soll den Integrationsprozess von Neuzugewanderten fördern und steuern und sich dabei primär auf die sozialpädagogische Begleitung der Integrationskurse fokussieren. Weiterhin fallen, den Förderrichtlinien zufolge, folgende Aufgaben in den Tätigkeitsbereich: „die Mitarbeit in kommunalen Netzwerken zur Förderung eines bedarfsgerechten Integrationsangebotes und die Mitwirkung bei der interkulturellen Öffnung der Regeldienste und Verwaltungsbehörden" (BMI 2010, S. 260). Damit sollen Sozialarbeiter_innen in der MBE qua Auftrag mit anderen Berufsgruppen zusammenarbeiten und Netzwerkarbeit betreiben.

Welche Akteur_innen sind in Netzwerken der Beratung für Geflüchtete vertreten und wie funktionieren diese Netzwerke? Wie kann multiprofessionelle Netzwerkarbeit in der praktischen Umsetzung gut gelingen? Wel- 


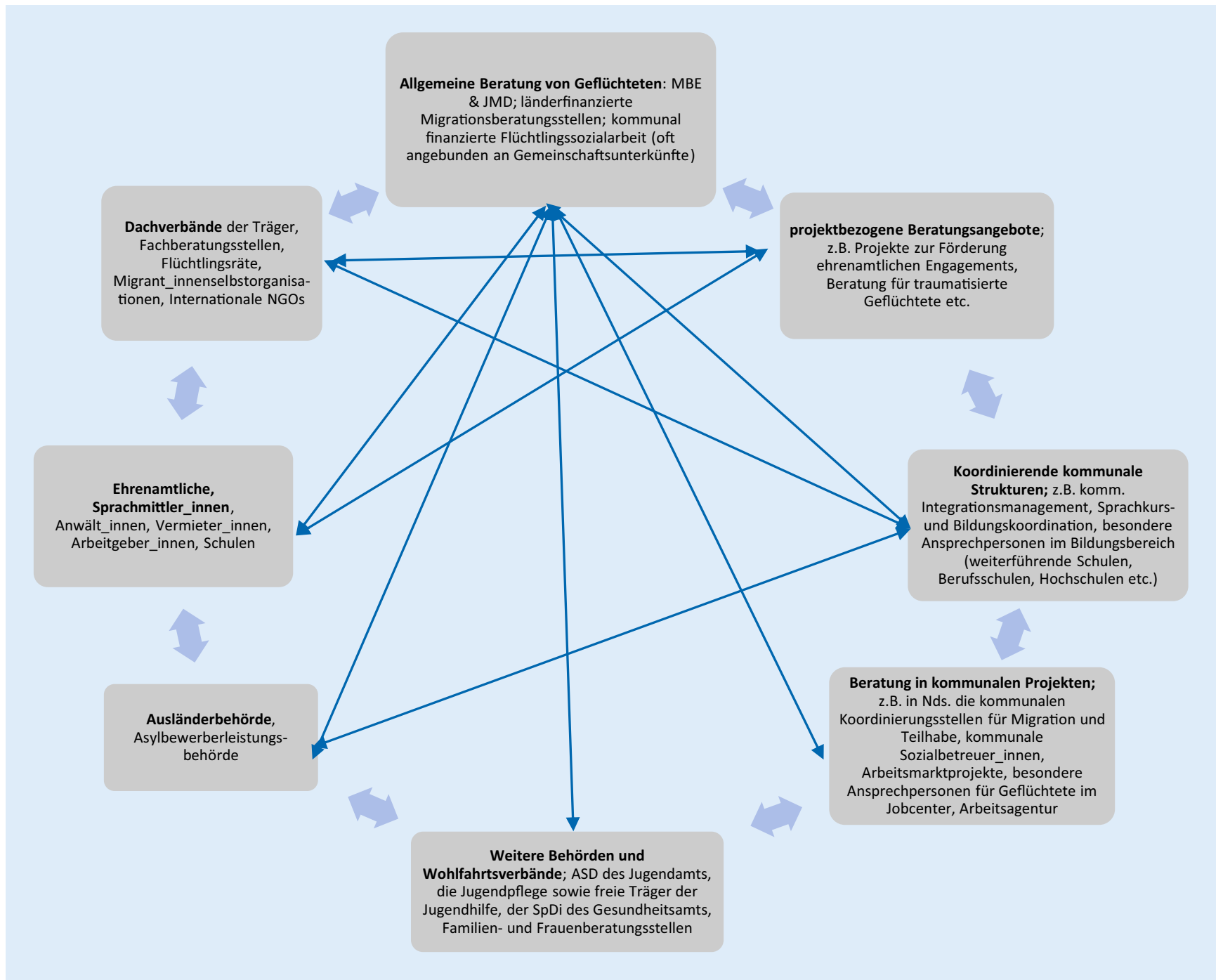

Abb. 1 Das multiprofessionelle Netzwerk der Migrationsberatung (Quelle: eigene Darstellung)

chen Herausforderungen begegnen Sozialarbeiter_innen in diesen Kontexten und wie können sie diese bewältigen? Entlang dieser Fragestellungen werden die Spezifika der multiprofessionellen Netzwerkarbeit in der Beratung von Geflüchteten herausgearbeitet und in Form eines Themenaufrisses aufgezeigt, welche wesentlichen Herausforderungen sich daraus ergeben und wie diesen aus Sicht der Sozialen Arbeit begegnet werden könnte.

\section{Das Netzwerk ist vielfältig und breit gefächert}

Das Netzwerk in der Beratung von Geflüchteten ist vielerorts außerordentlich multiprofessionell und breit gefächert aufgestellt. Die Vernetzung erfolgt meist nicht nur lokal, sondern auch (über)regional, landes- und bundesweit und sogar transnational, z. B. mit Einrichtungen von $\mathrm{IOM}^{2}$ und Fachstellen im Ausland. Das Schaubild zeigt die unterschiedlichen Akteur_innen, die entweder regelmäßig oder mehr punktuell in einem multiprofessionellen Netzwerk zusammenarbeiten (Abb. 1).
In der MBE, den Landesprogrammen und den projektförmigen Beratungsangeboten sind primär Sozialarbeiter_innen tätig, während die Beratung in kommunalen Koordinationsstellen von unterschiedlichen akademischen Berufsgruppen durchgeführt wird, da ein Studium der Sozialen Arbeit oder eine gleichwertige Qualifikation dort - anders als etwa im Bundesprogramm MBE - keine Voraussetzung zur Stellenbesetzung ist. In den Behörden sind, abgesehen vom Allgemeinen Sozialen Dienst (ASD) und Sozialpsychiatrischen Dienst (SpDi), meistens Verwaltungsfachleute die Ansprechpartner_innen; die Stellen von Sozialbetreuer_innen werden von unterschiedlichen persönlich qualifizierten Berufsgruppenvertreter_innen besetzt. Ehrenamtliche und Sprachmittler_innen haben wiederum völlig unterschiedliche berufliche Hintergründe, bei den Dachverbänden und Nichtregierungsorganisationen hingegen arbeiten überwiegend Sozialarbeiter_innen und Jurist_innen. 


\section{Sich auf gemeinsame Ziele einigen}

In der multiprofessionellen Zusammenarbeit in der Geflüchtetenberatung werden von den Akteur_innen durchaus nicht nur unterschiedliche, sondern manchmal auch gegensätzlich zueinander liegende Ziele formuliert. Darin zeigt sich eine erste Herausforderung in der multiprofessionellen Zusammenarbeit. Stellen wir uns einen Jugendlichen vor, der ohne seine Eltern aus Syrien geflohen ist und nun bei Verwandten lebt. Er besucht die 10. Klasse einer weiterführenden Schule und hat beim Jugendamt eine Vormundin. Das Ziel des beteiligten Klassenlehrers kann nun z. B. darin liegen, die schulischen Leistungen zu fördern, während seine Vormundin vor allem seine persönliche psychische und soziale Entwicklung in der Familie stärken möchte. Die Sozialarbeiter_in im JMD oder in der MBE versteht als dringendsten Wunsch des Jugendlichen die Zusammenführung mit seinen in einem Drittland lebenden Eltern und Geschwistern und will primär darauf hinarbeiten; der beteiligte Sozialbetreuer der Verwandten hält die Wohnsituation für das wichtigste Problem, da fünf Personen in drei Zimmern wohnen, und sorgt sich um die finanzielle Lage.

Eine Verständigung über die Priorisierung der unterschiedlichen Ziele bedingt zunächst eine Verständigung über unterschiedliche Systemlogiken und ,Normalitäten' (vgl. z. B. Bauer 2018). Danach kann ein gleichberechtigter Aushandlungsprozess zwischen den beteiligten Fachkräften unterschiedlicher beruflicher Hintergründe erfolgen. In multiprofessionellen Netzwerken ist die Zusammenarbeit oft „insbesondere dadurch charakterisiert, dass sich die Professionellen nur zu wenigen Zeitpunkten im Jahr zusammenfinden, um die gemeinsam zu leistenden Aufgaben zu besprechen“ (Bauer 2018, S. 734). Dabei erfolgt die Zusammenarbeit in der Regel punktuell, bei konkreten Einzelfällen und ist eine voraussetzungsvolle Aufgabe, da sich Zugänge und Zuständigkeiten sowie strukturelle Rahmenbedingungen (etwa bzgl. der Bezahlung und der Arbeitsbedingungen) der jeweiligen Arbeitsstelle unterscheiden. „Sie erfordert von den einzelnen Beteiligten die Fähigkeit, diese Aushandlungsprozesse mitzugestalten, die eigenen fachlichen Perspektiven und Positionen einzubringen, aber auch die der anderen Professionen wahrzunehmen, ohne sich diesen unterzuordnen." (Bauer 2018, S. 736) Eine gelingende multiprofessionelle Zusammenarbeit benötigt auf Seiten der Professionellen dafür vielfältige Fähigkeiten. Neben Respekt und Wertschätzung, Offenheit und Toleranz, Empathiefähigkeit, Klarheit im Auftreten, einer positiven lösungsorientierten Haltung, Konfliktfähigkeit u. a. (vgl. Kemp und Maar 2017) ist die Bereitschaft zur Übernahme von Verantwortung zentral. Als ein Ziel multiprofessioneller Zusammen- arbeit kann somit die „gemeinsame Verantwortungsübernahme“" (Bauer 2018, S. 736) bezeichnet werden, wobei Teilaufgaben i. d. R. auf die einzelnen Fachkräfte verteilt werden, um durch die gezielte Zusammenarbeit Problemstellungen umfassender und wirkungsvoller (vgl. Bauer 2018, S. 731) bearbeiten zu können. Gelingt dies, können die jeweilige Expertise, die Wissensbestände und Kompetenzen zum Vorteil der ratsuchenden Menschen zusammengeführt genutzt werden.

\section{Wer übernimmt welche Aufgaben?}

Die Zusammenarbeit mit Volljurist_innen soll dabei beispielhaft verdeutlichen, wie multiprofessionelle $\mathrm{Zu}$ sammenarbeit in der Beratung von Geflüchteten funktionieren kann. Das Rechtsdienstleistungsgesetz erfasst die Grenzen des Beratungshandelns für Sozialarbeiter_ innen und gilt für alle Beratungsbereiche mit rechtlichem Bezug. Sozialarbeiter_innen dürfen in begrenztem Rahmen außergerichtliche Tätigkeiten übernehmen (z. B. bei der Formulierung von Briefen unterstützen), sollen dabei aber selbst von Jurist_innen bei Bedarf unterstützt werden. Sollten die juristischen Wissensbestände der Beratungsfachkraft ausgeschöpft sein, ist die ratsuchende Person an eine Kanzlei oder eine andere Fachstelle weiter zu verweisen. Bevor dies notwendig ist, leisten Sozialarbeiter_innen in der Beratung zu einem großen Teil Aufklärungsarbeit, erklären, informieren und vermitteln, da den meisten Geflüchteten das deutsche Rechtssystem, die Strukturen, Institutionen und Verfahrenswege unbekannt sind (vgl. AWO 2018, S. 29 f.). Es geht darum, behördliche Schreiben und Bescheide zu erläutern und über Rechte und Pflichten aufzuklären. In der Zusammenarbeit mit Anwält_innen werden Sozialarbeiter_innen häufig geschätzt, da Ratsuchende sich an sie wenden, um anwaltliche Rechtsberatung und -vertretung vor- und nachzubereiten, denn Sozialarbeiter innen haben oftmals eine vertrauensvollere Beziehung zu den Ratsuchenden, einen intensiveren Kontakt (vgl. AWO 2018, S. 33) und genauere Kenntnis der Lebensumstände im Herkunftsland und der aktuellen Lebenssituation. Zudem kennen sie auch die flankierenden Problemlagen wie finanzielle Engpässe, Verschuldung und familiäre Probleme, welche das rechtliche Problem beeinflussen und überlagern können.

Hier können Sozialarbeiter_innen im Auftrag von und mit Einverständnis der Ratsuchenden zwischen Anwält_innen und Ratsuchenden vermitteln und die Verständigung begleiten. Auf der einen Seite sind Sozialarbeiter_innen durch Kenntnisse über die Strukturen in den Herkunftsländern sowie durch die Vernetzung etwa mit Sprachmittler_innen und Organisationen wie IOM in der Lage, komplizierte Verfahren etwa im Bereich 
des Familiennachzugs mit zu unterstützen und den langwierigen und oft sehr belastenden Prozess auch psychosozial zu begleiten. Auf der anderen Seite haben Volljurist_innen die Kompetenz, Sozialarbeiter_innen und Geflüchtete bei komplexer Rechtslage zu informieren und zu beraten. Durch die strukturell gegebene Abgrenzung der Aufgabenbereiche wird die multiprofessionelle Zusammenarbeit an dieser Stelle erleichtert und stellt eine große Ressource für die gemeinsame Unterstützung der geflüchteten Menschen dar.

Ein weiteres Beispiel für die Charakteristika multiprofessioneller Zusammenarbeit ist die Kooperation mit kommunalen Sozialbetreuer_innen. Diese haben zumeist die Aufgabe, Geflüchtete bei der Wohnungssuche und -ausstattung zu unterstützen und bei Fragen des alltäglichen Lebens (z. B. Mülltrennung) zur Seite zu stehen sowie Behördengänge zu begleiten. Sozialarbeiter_innen in der Beratung können dieses i.d. R. nicht leisten. Allein schon aufgrund der hohen Zahl der Ratsuchenden ist eine Begleitung des Einzelfalls schwer möglich. Gerade in der Zusammenarbeit mit weiteren Akteur_innen, besonders in den Behörden, ist eine Abstimmung zwischen Sozialbetreuer_innen und Sozialarbeiter_innen, die denselben Einzelfall betreuen, essentiell wichtig. Gelingt diese, lässt es sich vermeiden, dass eine Aufgabe von zwei Bearbeitenden unnötigerweise ausgeführt wird. Auch Missverständnissen kann so vorgebeugt werden und Ziele vereinbart werden, bei denen je nach Qualifikation und Auftrag verschiedene, sich ergänzende Aufgaben übernommen werden. Ähnliches gilt für die Zusammenarbeit mit Ehrenamtlichen und Berater_innen kommunaler Koordinierungsstellen.

\section{Gute Netzwerkarbeit braucht Ressourcen}

Absprachen benötigen neben Verständnis und Wertschätzung unterschiedlicher Sichtweisen und Logiken auch Zeit und Raum: „Auf team-, organisations- und netzwerkbezogener Ebene sind zeitliche, sachliche und soziale Ressourcen unabdingbar, um eine produktive Zusammenarbeit überhaupt zu ermöglichen. “ (Bauer 2018, S. 736)

Wohlfahrtsverbände haben in den vergangenen Jahren mehrfach darauf hingewiesen, dass die zur Verfügung gestellten Mittel etwa für das Bundesprogramm MBE/ JMD vor dem Hintergrund des wachsenden Bedarfs und der Ausweitung der Zielgruppe nicht ausreichen (BAGFW 2018). Zudem fordern sie die Finanzierung einer bundesgeförderten, qualifizierten und behördenunabhängigen Asylverfahrensberatung durch die Verbände der Freien Wohlfahrtspflege (nach $\$ 12$ a Asylgesetz) ${ }^{3}$. Bei der Verfügbarkeit konkreter allgemeiner sozialer Beratungsangebote für Geflüchtete zeigen sich regional ek- latante Unterschiede, da ein großer Teil der Beratungsstellen projektfinanziert ist und die Beantragung und Stellenbesetzung damit von den vor Ort vorhandenen Trägern abhängt. In manchen Kreisstädten gibt es nur eine halbe Personalstelle für die allgemeine soziale Beratung aller Geflüchteten in der Stadt, in anderen ein halbes Dutzend. Da keine amtliche Statistik über das Verhältnis der Zahl von (potentiellen) Ratsuchenden in einer Kommune zur Zahl aller vorhandenen Beratungsangebote geführt wird, kann hier nur auf Erfahrungswerte und Eindrücke aus der Praxis verwiesen werden. ${ }^{4}$ In Großstädten ist die Infrastruktur tendenziell wesentlich ausdifferenzierter und dichter. Zudem verändert sich die Verfügbarkeit von örtlichen Beratungsangeboten, wenn Mittel des Vorjahres im darauffolgenden Jahr nicht mehr zur Verfügung stehen und damit Beratungsstellen geschlossen werden. Die Akteur_innen des Netzwerks sind damit zu einem Anteil instabil, was den Aufbau und die Entwicklung von Netzwerken schwächt, da diese auch durch persönliche Beziehungen und Vertrauen getragen werden.

Ein weiteres Problem entsteht auch durch den Einbezug bzw. Ausschluss bestimmter Geflüchtetengruppen etwa genau dann, wenn in einer Kommune eine Beratungsstelle „für alle“ Geflüchteten geschlossen wird oder zeitweise unbesetzt ist. So fallen gerade die Asylsuchenden, bei denen kein dauerhafter und rechtmäßiger Aufenthalt zu erwarten ist, vielerorts durch das Raster des vorhandenen Beratungsangebots. Ist dies der Fall, können sie weder in Bezug auf ihr Asylverfahren noch in anderen Lebensbereichen angemessen vor Ort beraten werden. In ländlichen Räumen mutet der Verweis auf die nächstliegende Großstadt angesichts der häufig prekären finanziellen Lage der Menschen zynisch an. Dieses Schlaglicht auf eine Untergruppe innerhalb der heterogenen Gesamtgruppe der Geflüchteten insgesamt macht deutlich, dass ausreichende personelle Ressourcen vielerorts nicht vorhanden sind. Diese hier beleuchtete Situation des Ressourcenmangels führt zu einer starken Mehrbelastung der vorhandenen Beratungsstellen. Während die BAGFW (2018) ausführt, dass eine Vollzeit MBE Stelle durchschnittlich ca. 150 Klient_innen plus Angehöriger berät, sind die Fallzahlen mancherorts teilweise doppelt so hoch (vgl. Bugaj-Wolfram 2018). Ratsuchende wenden sich in ihrer Not an Regeldienste, die für komplexe aufenthaltsrechtliche Fragestellungen (z. B. Familiennachzug) oder auch für fluchtspezifische psychosoziale Problemlagen selten ausreichend weitergebildet sind.

Angesichts dessen sind Sozialarbeiter_innen in der Geflüchtetenberatung vor eine sehr große Herausforderung gestellt, neben der konkreten Beratung im Einzelfall zeitliche Ressourcen für die Abstimmungs- und Aushandlungsprozesse im Netzwerk abzustellen. 


\section{Die Rolle der Sozialen Arbeit}

Im multiprofessionellen Netzwerk für die Unterstützung geflüchteter Menschen hat Soziale Arbeit eine besondere Rolle, weil sie sich von anderen Berufsgruppen auch durch ihre Professionsethik unterscheidet. Allgemein zeichnet sich professionelles Handeln dadurch aus, dass es dem „Ziel dient, die physische, soziale, seelische und rechtliche Integrität von Menschen zu gewährleisten " (Bauer 2018, S. 729). Soziale Arbeit hat zudem den Anspruch, sich an international vereinbarten ethischen Standards zu orientieren, die sich im Kern auf die Würde des Menschen, auf universelle Menschenrechte und soziale Gerechtigkeit beziehen (vgl. IASSW 2018). Stoßen Sozialarbeiter_innen in einer kooperativen Zusammenarbeit mit etwa der Ausländerbehörde oder dem Jobcenter auf eine institutionelle Logik, die diskriminierende Effekte auf den einzelnen Menschen hat und wird dies von dem/der Behördenmitarbeiter_in nicht in Frage gestellt $^{5}$, kann ein ethischer Konflikt entstehen. Der DBSH fordert in einem Positionspapier (2019) die Soziale Arbeit auf, gegen menschenfeindliches und diskriminierendes Handeln einzutreten und „potentielle Opfer vor einer entsprechenden Politik zu schützen bzw. eine solche Politik entsprechend aufzudecken und zu kritisieren.“ Dem eigenen professionellen Selbstverständnis folgend sind Sozialarbeiter_innen aufgefordert, an dieser Stelle anwaltschaftlich für die Ratsuchenden einzutreten und können dadurch in einen Interessens- und Zielkonflikt geraten. Das Verstehen der unterschiedlichen Selbstverständnisse und Zuständigkeiten ist dabei die aus meiner Sicht zentrale Kompetenz. Auf Seiten der Behördenmitarbeiter_innen kann der Eindruck entstehen, Sozialarbeiter_innen seien „,idealistisch“ und hätten keine ausreichende Kenntnis der rechtlichen Regelungen, auf die sich die Behörde beziehe und beziehen müsse. Gerade aus diesem Grund braucht es gute Kenntnisse der Gesetze und Ausführungsbestimmungen auf Seiten der Sozialarbeiter_innen, um einen Dialog auf Augenhöhe führen zu können. Die Perspektive aus der Beratungsarbeit, das Wissen über den „ganzen Fall“, über die persönlichen und familiären Hintergründe, können dann viel eher zum Vorteil der Ratsuchenden in die Verständigung mit den Behördenmitarbeiter_innen eingebracht werden. Der regelmäßige Austausch, das Aufeinander zugehen, der Aufbau regelmäßiger Netzwerktreffen ist dabei der Schlüssel zu einer gelingenden Zusammenarbeit trotz unterschiedlicher Sichtweisen und Zuständigkeiten. Dabei ist die andauernde Reflexion von Asymmetrien auf der Ebene struktureller Macht unabdingbar (vgl. Kraus und Krieger 2016). Diese können aber weniger auf der Ebene zwischen zwei Personen in unterschiedlichen Institutionen bearbeitet und gelöst werden, sondern viel- mehr durch die Stärkung der Interessen Geflüchteter auf einer gesamtgesellschaftlichen und politischen Ebene. Ein konkreter Beitrag ist die Solidarisierung der Berufsorganisationen und Dachverbände Sozialer Arbeit mit Migrant_innenselbstorganisationen und Flüchtlingsräten, um für eine humanere Migrationspolitik und für eine Verbesserung der Unterstützungsstrukturen für Geflüchtete einzutreten. Daneben muss die Interkulturelle Öffnung der Behörden und Regeldienste weiter ausgebaut werden, damit vorhandene Angebote auch faktisch nutzbar sind und so alle hier lebenden Menschen ihre Rechte angemessen wahrnehmen können. Ein Beitrag dazu ist die Zusammenarbeit zwischen den unterschiedlichen Berufsgruppen im Netzwerk der Migrationsberatung. Gelingt diese, kann sie für alle Beteiligten effektiv und hilfreich sein und so zur Verbesserung der Lebenssituation von ratsuchenden Menschen beitragen.

1. BAMF gefördertes Beratungsangebot für Jugendliche und junge Erwachsene bis 27 Jahre.

2. Die Internationale Organisation für Migration (IOM) ist eine weltweite zwischenstaatliche Organisation im UN-System, die u. a. in Kooperation mit den deutschen Botschaften die Familienzusammenführung unterstützt.

3. Mit der Verabschiedung des Bundeshaushalts für das Jahr 2020 wurde deutlich, dass diese Forderung nicht umgesetzt werden soll, was von Flüchtlingsorganisationen und Fachverbänden scharf kritisiert wird. So weist Pro Asyl (2019) darauf hin, dass die „Beteiligung von Wohlfahrtsverbänden und anderen Trägern [.] nur als Option angelegt und laut Gesetz [.] nur die Übernahme von Sachkosten vorgesehen" ist. Somit fehlen die entscheidenden Bundesgelder für Personalkosten und eine unabhängige flächendeckende Verfahrensberatung ist damit schlicht nicht umsetzbar.

4. Insofern beziehe ich mich auf persönliche Eindrücke aus dem Austausch mit Migrationsberater_innen und Flüchtlingssozialarbeiter_innen auf landes- und bundesweiten Tagungen zwischen 2016 und 2019.

5. Dies ist z. B. dann der Fall, wenn ein_e Geflüchtete_r eine bestimmte Fördermaßnahme (z. B. einen besonderen Sprachkurs) nicht bekommt, weil er/sie aus einem bestimmten Herkunftsland kommt oder vor/nach einem bestimmten Datum eingereist ist o. ä., wodurch zum entscheidenden Zeitpunkt nicht (mehr) von einer „guten Bleibeperspektive" ausgegangen wird.

Funding. Open Access funding provided by Projekt DEAL.

Open Access. Dieser Artikel wird unter der Creative Commons Namensnennung 4.0 International Lizenz veröffentlicht, welche die Nutzung, Vervielfältigung, Bearbeitung, Verbreitung und Wiedergabe in jeglichem Medium und Format erlaubt, sofern Sie den/die ursprünglichen Autor(en) und die Quelle ordnungsgemäß nennen, einen Link zur Creative Commons Lizenz beifügen und angeben, ob Änderungen vorgenommen wurden.

Die in diesem Artikel enthaltenen Bilder und sonstiges Drittmaterial unterliegen ebenfalls der genannten Creative Commons Lizenz, sofern sich aus der Abbildungslegende nichts anderes ergibt. So- 
fern das betreffende Material nicht unter der genannten Creative Commons Lizenz steht und die betreffende Handlung nicht nach gesetzlichen Vorschriften erlaubt ist, ist für die oben aufgeführten Weiterverwendungen des Materials die Einwilligung des jeweiligen Rechteinhabers einzuholen.

Weitere Details zur Lizenz entnehmen Sie bitte der Lizenzinformation auf http://creativecommons.org/licenses/by/4.0/deed.de

\section{Literatur}

AWO Bundesverband e. V. (2018). Soziale Arbeit mit geflüchteten Menschen. Ein Leitfaden für die AWO Beratungspraxis. https://www.awo.org/ soziale-arbeit-mit-gefluechteten-menschen-ein-leitfaden-fuer-die-awoberatungspraxis-0. Zugegriffen: 3. März 2020.

BAGFW (2018). Pressemitteilung: Migrationsberatung für erwachsene Zuwanderer - finanzielle Ausstattung reicht noch immer nicht aus. https:/www.bagfw.de/veroeffentlichungen/stellungnahmen/positionen/ detail/migrationsberatung-fuer-erwachsene-zuwanderer-finanzielleausstattung-reicht-noch-immer-nicht-aus. Zugegriffen: 3. März 2020

Bauer, P. (2018). Multiprofessionalität. In G. Graßhoff, A. Renker \& W. Schröer (Hrsg.), Soziale Arbeit - eine elementare Einführung (S. 727-739). Wiesbaden: Springer.

BMI (2010). Förderrichtlinien zur Durchführung einer Migrationsberatung für Erwachsene (MBE). In $G M B l 2010$ (S. 260). Geändert durch Verwaltungsvorschrift vom 15.06.2016 (GMBl 2016, Nr. 28, S. 548).

Bugaj-Wolfram, N. (2018in). Wortbeitrag des Paritätischen Gesamtverbands bei dem MBE-Aktionstag 2018 in Berlin zur Bedeutung der MBE im Integrationsprozess und zur Notwendigkeit der Verbesserung von Rahmenbedingungen.www.der-paritaetische.de/fileadmin/user_upload/ Schwerpunkte/Migration/MBE-Âktionstag_2018/Aktionstag_Beitrag.pdf. Zugegriffen: 3. März 2020.

DBSH (2019). Positionspapier „DBSH verurteilt menschenfeindliches und diskriminierendes Handeln ". https://www.dbsh.de/der-dbsh/dbshmitteilungen/detail/positionspapier-zu-dbsh-verurteilt-menschenfeindlichesund-diskriminierendes-handeln.html. Zugegriffen: 3. März 2020.

IASSW (2018). Global Social Work Statement of Ethical Principles (GSWSEP). www.iassw-aiets.org/wp-content/uploads/2018/04/Global-SocialWork-Statement-of-Ethical-Principles-IASSW-27-April-2018-1.pdf. Zugegriffen: 3. März 2020.

Kemp, M. \& Maar, K. (2017). Professionalität Sozialer Arbeit im multiprofessionellem Setting. Vortrag Fachtag FH Köln am 11.05.2017. https:// www.th-koeln.de/mam/downloads/deutsch/hochschule/aktuell/nachrichten/ f01/folien_ag_b_kemp_maar.pdf. Zugegriffen: 3. März 2020.

Kraus, B., \& Krieger, W. (Hrsg.). (2016). Macht in der Sozialen Arbeit. Interaktionsverhältnisse zwischen Kontrolle, Partizipation und Freisetzung. Lage: Jacobs.

Ministerium für Soziales, Gesundheit und Gleichstellung (2017). Richtlinie über die Gewährung von Zuwendungen zur Förderung der Migrationsberatung in Niedersachsen (Richtlinie Migrationsberatung). Erl. d. MS v. 14. 7. 2017 - 301.31-04011-04

PRO ASYL (2019). Pressemitteilung: Bundeshaushalt: PRO ASYL prangert Mogelpackung bei der Asylverfahrensberatung an. https:// www.proasyl.de/pressemitteilung/bundeshaushalt-pro-asyl-prangertmogelpackung-bei-der-asylverfahrensberatung-an/. Zugegriffen: 3. März 2020 . 